\title{
Myocardial protection from ischemia/reperfusion injury by endogenous and exogenous HGF
}

\author{
Teruya Nakamura,,${ }^{1,2}$ Shinya Mizuno, ${ }^{1}$ Kunio Matsumoto, ${ }^{1}$ Yoshiki Sawa, ${ }^{2}$ \\ Hikaru Matsuda, ${ }^{2}$ and Toshikazu Nakamura ${ }^{1}$ \\ ${ }^{1}$ Division of Biochemistry, Department of Oncology, Biomedical Research Center B7, and \\ ${ }^{2}$ Department of Surgery, Course of Interventional Medicine E1, Osaka University Graduate School of Medicine, Suita, Japan
}

Address correspondence to: Toshikazu Nakamura, Division of Biochemistry, Department of Oncology,

Biomedical Research Center B7, Osaka University Graduate School of Medicine, 2-2 Yamadaoka, Suita 565-0871, Japan.

Phone: 81-6-6879-3783; Fax: 81-6-6879-3789; E-mail: nakamura@onbich.med.osaka-u.ac.jp.

Received for publication May 2, 2000, and accepted in revised form November 6, 2000.

\begin{abstract}
Using a rat model of ischemia/reperfusion injury, we demonstrate here that HGF is cardioprotective due to its antiapoptotic effect on cardiomyocytes. Following transient myocardial ischemia and reperfusion, c-Met/HGF receptor expression rapidly increased in the ischemic myocardium, an event accompanied by a dramatic increase in plasma HGF levels in the infarcted rats. When endogenous HGF was neutralized with a specific antibody, the number of myocyte cell deaths increased markedly, the infarct area expanded, and the mortality increased to $50 \%$, as compared with a control group in which there was no mortality. Plasma from the myocardial infarcted rats had cardioprotective effects on primary cultured cardiomyocytes, but these effects were significantly diminished by neutralizing HGF. In contrast, recombinant HGF administration reduced the size of infarct area and improved cardiac function by suppressing apoptosis in cardiomyocytes. HGF rapidly augmented Bcl-xL expression in injured cardiomyocytes both in vitro and in vivo. As apoptosis of cardiomyocytes is one of the major contributors to the pathogenesis in subjects with ischemia/reperfusion injury, prevention of apoptosis may prove to be a reasonable therapeutic strategy. Supplements of HGF, an endogenous cardioprotective factor, may be found clinically suitable in treating subjects with myocardial infarction.
\end{abstract}

J. Clin. Invest. 106:1511-1519 (2000).

\section{Introduction}

During each of several recent years, more than 6 million people worldwide died of ischemic heart disease, and ischemic heart disease is predicted to be the leading cause of individual deaths the world over in the near future (1). Although treatments for ischemic heart disease such as recanalization therapy have progressed, reperfusion treatment often makes way for myocardial injury by increasing inflammatory responses. There is a growing body of evidence that apoptosis of cardiomyocytes is one of the major contributors to myocardial infarction and to ischemia/reperfusion injury (2-4). As apoptosis occurs within 24 hours and induces massive or submassive losses of myocytes, the susceptibility to cardiac dysfunction increases (5). Therefore, if cardiomyocyte apoptosis could be inhibited, cardiac pathophysiology and dysfunction due to myocardial infarction and ischemia/reperfusion injury could be minimized.

HGF, originally purified and cloned as a potent mitogen for hepatocytes $(6,7)$, has mitogenic, motogenic, morphogenic, and antiapoptotic activities in various cell types (8-12). Pluripotent activities of HGF are mediated by a membrane-spanning tyrosine kinase receptor encoded by the $c$-met proto-oncogene $(13,14)$. Physiologically, HGF plays a role as an organotrophic factor for regeneration and protection, including the liver $(15,16)$, kidney $(17,18)$, and lung $(19,20)$. Although anti-cell death actions of HGF were initially implicated by findings that laboratory animals given HGF after acute induced hepatic or renal injury had much less histological damage and more satisfactory organ functions, subsequent studies extended this anti-cell death activity of HGF to a variety of cells, including renal epithelial cells and neurons $(21,22)$, as well as hepatocytes $(23,24)$.

Previous studies showed that HGF and c-Met mRNA were transiently upregulated in the myocardium during heart development in mice (25) and plasma levels of HGF were markedly elevated in patients with acute myocardial infarction (26). We recently reported that HGF gene transfection into the myocardium attenuated ischemia/reperfusion injury in isolated perfused rat hearts (27); however, whether HGF plays a cardiotrophic role in pathophysiological conditions and has therapeutic potential in cardiac ischemia/reperfusion injury remained to be addressed. Using two distinct approaches, biological neutralization of endogenous HGF and supplement of recombinant HGF, we now provide evidence that endogenous HGF is cardioprotective and exogenous HGF attenuates ischemia/reperfusion injury by directly protecting cardiomyocytes. 


\section{Methods}

Ischemia/reperfusion injury model. Myocardial ischemia/reperfusion injury was induced, using 4week-old male Sprague-Dawley rats, as described (28). The left coronary artery was ligated at the point $2 \mathrm{~mm}$ distal from the ascending aorta. After 20 minutes of occlusion, the ligature was released and blood flow was visualized. Forty-eight hours after reperfusion, the right carotid artery was cannulated with a microtip catheter and the catheter was advanced into the left ventricle (LV) to measure blood pressure. All animal experiments were done in accordance with NIH guidelines, as dictated by the Animal Care Facility at Osaka University Graduate School of Medicine.

Real-time quantitative RT-PCR. Total RNA was prepared from the myocardial tissue, using TRIzol (Life Technologies Inc., Rockville, Maryland, USA). One $\mu \mathrm{g}$ of total RNA was reverse-transcribed into first strand cDNA with random hexaprimer using Superscript II reverse transcriptase (Life Technologies Inc.). Quantitative PCR was performed as described (29). Sequences for primers and TaqMan fluorogenic probes (PerkinElmer Biosystems, Foster City, California, USA) were as follows: c-Met, forward primer, $5^{\prime}$-GTA CGG TGT CTC CAG CAT TTT T-3', reverse primer, $5^{\prime}$-AGA GCA CCA CCT GCA TGA AG-3', probe, 5'(FAM)-ACC ACG AGC ACT GTT TCA ATA GGA CCC-(TAMRA)3'; GAPDH, forward primer, $5^{\prime}$-CCA TCA CTG CCA CTC AGA AGA C-3', reverse primer, 5'-TCA TAC TTG GCA GGT TTC TCC A-3', probe, 5'(FAM)-CGT GTT CCT ACC CCC AAT GTA TCC GT (TAMRA) 3'. Experimental samples were matched to a standard curve generated by amplifying serially diluted products, using the same PCR protocol. To correct for variability in RNA recovery and efficiency of reverse transcription, GAPDH cDNA was amplified and quantitated in each CDNA preparation.

Recombinant HGF, anti-rat HGF antibody, and biochemical analysis. Human or rat recombinant HGF was purified from culture media of Chinese hamster ovary cells transfected with an expression vector containing human or rat HGF cDNA, respectively, as described (7). The purity of HGF was $>98 \%$, as determined by SDSPAGE. Anti-rat HGF neutralizing antibody was prepared as described elsewhere $(30,31)$, and the IgG fraction was purified on a protein A-Sepharose column (Pharmacia Biotech AB, Uppsala, Sweden). In cell scattering assay with Madin-Darby canine kidney cells (an authentic bioassay for HGF), $5 \mu \mathrm{g} / \mathrm{ml}$ of anti-rat HGF IgG completely inhibited cell scattering induced by 5 $\mathrm{ng} / \mathrm{ml}$ rat $\mathrm{HGF}$, but not that induced by human HGF (data not shown). Likewise, in cultured rat cardiomyocytes, this antibody inhibited extracellular signal-regulated kinase (ERK) activation by rat HGF, but not that by IGF-1, leukemia inhibitory factor, and bFGF (data not shown). Moreover, no significant effects were seen on survival, and no histological abnormalities were seen in the liver, kidney, lung, and heart in the animals when this antibody was administered into sham-operated rats (data not shown). HGF levels and creatine phosphokinase (CPK) levels were measured using rat HGF ELISA kits (Institute of Immunology, Tokyo, Japan) and Wako CPK test kits (Wako Pure Chemicals Industries, Osaka, Japan), respectively.

Histological analysis and terminal deoxynucleotidyl transferase-mediated dUTP nick end-labeling assay. The LV from each heart was excised, sliced transversely $4 \mathrm{~mm}$ basal from the apex, fixed in $10 \%$ formalin in PBS, and embedded in paraffin. Evaluation of the infarct area was done, as described (32) but with some modifications. In brief, transverse sections were labeled with monoclonal $\alpha$-sarcomeric actin (clone 5C5, Sigma Chemical Co., St. Louis, Missouri, USA) followed by visualization using a kit for alkaline phosphatase (DAKO Corp., Carpinteria, California, USA). Ratio of the nonviable area (unlabeled with $\alpha$-sarcomeric actin antibody) to the area of the whole LV was analyzed for each section. For double immunohistochemistry of $\alpha$-sarcomeric actin and c-Met, the slides were incubated overnight with anti- $\alpha$-sarcomeric actin antibody and polyclonal anti-mouse c-Met (SP 260; Santa Cruz Biotechnology Inc., Santa Cruz, California, USA), respectively. The slides were then sequentially incubated with rhodamin-conjugated anti-mouse IgG (ICN Pharmaceuticals Inc., Aurora, Ohio, USA) and FITC-conjugated anti-rabbit IgG (ICN Pharmaceuticals Inc.), respectively. As a control staining, c-Met antibody was absorbed prior to staining with an excess amount of the specific peptide. For immunohistochemical detection of Bcl-xL, the slides were incubated with polyclonal anti-mouse Bcl-xL (S-18; Santa Cruz Biotechnology Inc.) for 1 hour, followed by sequential incubation with biotinylated goat anti-rabbit IgG (Vector Laboratories, Burlingame, California, USA) and peroxidase-labeled avidin-biotin complex, using a kit (VECTASTAIN Elite ABC; Vector Laboratories). Terminal deoxynucleotidyl transferase-mediated dUTP nick end-labeling (TUNEL) staining was done using an In Situ Apoptosis Detection Kit (MK-500; Takara Shuzo Co., Tokyo, Japan). The number of TUNEL- or Bcl-xL-positive cardiomyocytes was counted at a 400 -fold field magnification, using at least ten randomly selected fields per section.

Cell culture and survival assay. Primary cultures of neonatal rat cardiomyocytes were prepared using methods originally described by Simpson et al. (33), but with minor modification. Purity of the isolated cardiomyocytes exceeded $90 \%$ at the time of each experiment, as determined by $\alpha$-sarcomeric actin immunolabeling. In survival assays, cells were seeded on 96-well culture dishes at a cell density of $2 \times 10^{4} / \mathrm{cm}^{2}$. After 2 days of culture in DMEM containing 10\% FBS, cells were cultured in serum-free medium for 24 hours. HGF or vehicle alone was added 1 hour prior to $\mathrm{H}_{2} \mathrm{O}_{2}$ treatment, and $50 \mu \mathrm{M} \mathrm{H}_{2} \mathrm{O}_{2}$ was added for 1 hour followed by 6 hours of culture. The viability of the cells was assessed, using WST-1 assay kits (Dojindo Co., Tokyo, Japan), an advanced MTT assay. Since the MTT/WST1 assay is based on formazan reaction only by metabolic active cells, it detects both apoptotic and necrotic cell death. The survival of cells, in percent, was defined as 
(absorbance in each well) / (absorbance in a control well) $\times 100(\%)$. To test the effect of rat plasma on cardioprotection, cardiomyocytes were cultured for 2 hours in the presence or absence of $10 \%$ plasma from normal rats or rats with myocardial infarction. Immediately before use, the plasma was preincubated for 2 hours with normal rabbit IgG or anti-rat HGF IgG. The cells were then treated with $50 \mu \mathrm{M} \mathrm{H}_{2} \mathrm{O}_{2}$ for 1 hour and further cultured for 6 hours.

ERK-1/2 activity and Western blots. After serum starvation for 60 hours, cardiomyocytes were treated with 10 $\mathrm{ng} / \mathrm{ml} \mathrm{HGF}$ for 10 minutes and lysed with SDS sample buffer. Cell lysate was subjected to SDS-PAGE on a $12 \%$ polyacrylamide gel, and proteins were electroblotted on PVDF membrane (Bio-Rad Laboratories Inc., Hercules, California, USA). After blocking, the membrane was sequentially incubated with anti-phospho-p44/p42 mitogen-activated protein kinase (ERK$1 / 2$ ) antibody (E10; New England BioLabs Inc., Beverly, Massachusetts, USA), biotinylated anti-mouse IgG (Vector Laboratories), horseradish peroxidase-conjugated streptavidin (Amersham Pharmacia Biotech UK, Little Chalfont, United Kingdom), and an enhanced chemiluminescence reagent (Amersham Pharmacia Biotech UK). To detect total ERK protein, the cell lysate was subjected to Western blot, as above except for use of an anti-ERK-1 antibody (K-23; Santa Cruz Biotechnology Inc.). To detect c-Met protein, cardiomyocytes were transferred to serum-free DMEM with or without $50 \mu \mathrm{M} \mathrm{H}_{2} \mathrm{O}_{2}$ and cultured for 24 hours. Cells were lysed in SDS sample buffer, the cell lysate was subjected to SDS-PAGE on a 7.5\% polyacrylamide gel, and proteins were electroblotted on PVDF membrane. The membrane was subjected to overnight blocking and sequentially immunoblotted, using an anti-mouse c-Met polyclonal antibody. To examine specificity of the antibody to HGF, plasma obtained from rats with ischemia/reperfusion injury was immunoprecipitated with anti-HGF antibody or normal rabbit IgG for 2 hours, and the immunoprecipitate absorbed to protein A-Sepharose was subjected to SDS-PAGE under nonreducing conditions. Immunoblotting was done, using biotinylated anti-rat

\section{Figure 1}

Changes in pathophysiology and HGF/c-Met expression in rats with ischemia/reperfusion injury. (a-d) Histopathology of the heart. At 6 hours after treatment, infiltration with inflammatory cells was seen in the LVFW (a, b). At 24 hours after ischemia/reperfusion, a more diffuse infiltration of inflammatory cells was evident (c), and the infarct lesion had expanded widely at 48 hours (d) Bars: a, c, and d, $200 \mu \mathrm{m} ; \mathbf{b}, 50 \mu \mathrm{m}$. (e) Change in CPK activity in sera. $I / R$, ischemia/reperfusion injury. ${ }^{A} P<0.001,{ }^{B} P<0.01$, ${ }^{C} P<0.05$. (f) Plasma HGF levels determined by ELISA. ${ }^{A} P<0.001,{ }^{B} P<0.01$, ${ }^{C} P<0.05$. (g) Change in c-Met mRNA expression in myocardium, as determined by quantitative RT-PCR. ${ }^{A} P<0.001,{ }^{B} P<0.01,{ }^{C} P<0.05$ compared with IVS; $D P<0.001$, ${ }^{P}<0.01$ compared with sham LV. (h-m) Immunohistochemical findings for c-Met. (h-k) Double immunohistochemistry for $\alpha$-sarcomeric actin and c-Met in the heart resected 48 hours after reperfusion. Photographs of the LVFW (h, i) and IVS (j, k) of a section are shown. Red $(\mathbf{h}, \mathbf{j})$ and green $(\mathbf{i}, \mathbf{k})$ fluorescence, respectively, indicate $\alpha$-sarcomeric actin and c-Met labeling. (I and $\mathbf{m})$ Immunostaining for cMet in the sham-operated myocardium (I) and the border region between infarcted and noninfarcted area ( $\mathbf{m})$. To detect background staining, anti-c-Met antibody was preabsorbed with antigenic synthetic peptide in a serial section (inset in $\mathbf{m}$ ). Bars: $100 \mu \mathrm{m}$.
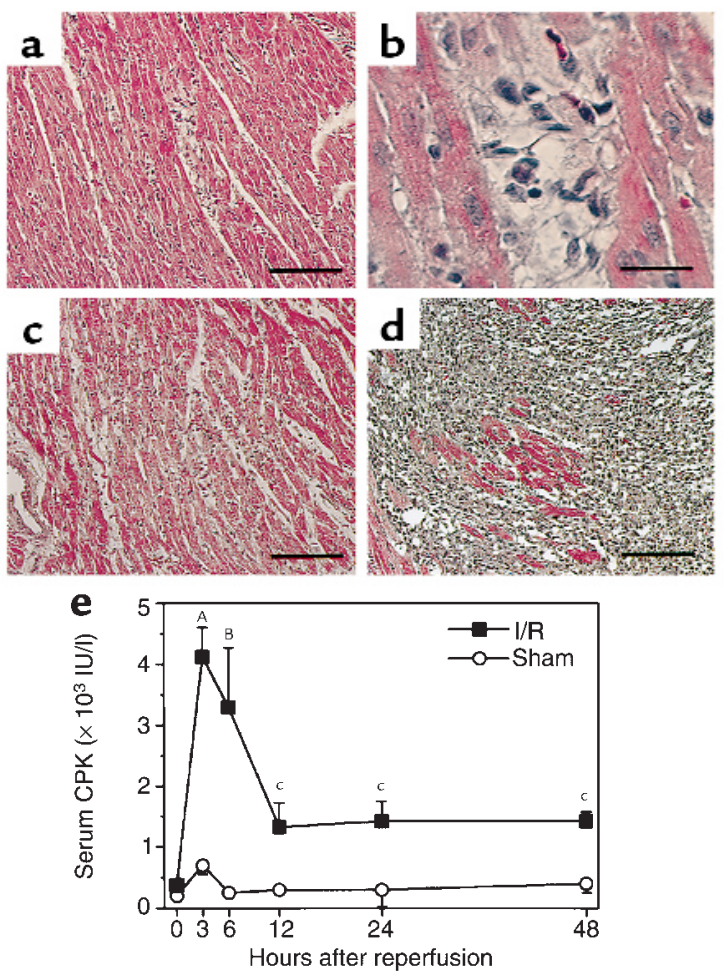

f
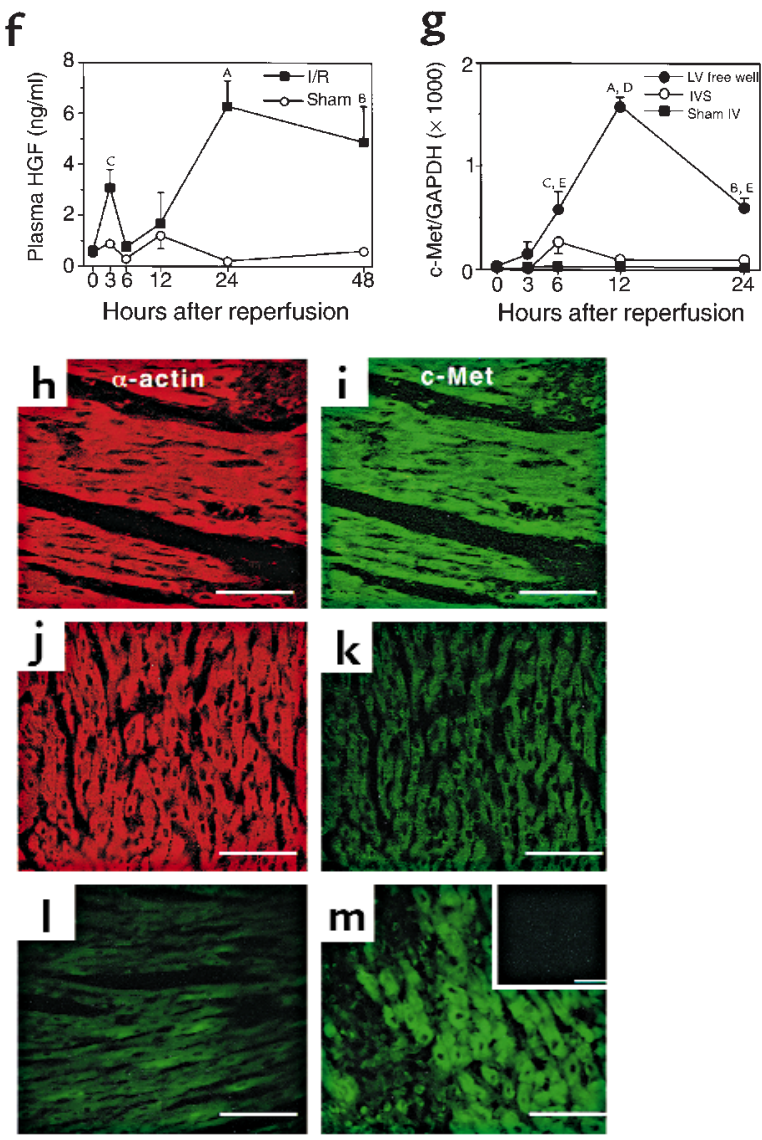
HGF antibody as a primary antibody. To detect Bcl-xL protein, the cell lysate or tissue extract was subjected to SDS-PAGE, and immunoblotting was done with polyclonal anti-mouse Bcl-xL, as described above.

Statistical analysis. We used Student's unpaired $t$ test or ANOVA to determine the statistical significance. Survival rates were compared by Kaplan-Meier method followed by log-rank test. $P<0.05$ was considered significant.

\section{Results}

Changes in pathophysiology and expression of $\mathrm{HGF} / \mathrm{c}-\mathrm{Met}$ in a rat model of ischemia/reperfusion injury. Six hours after transient occlusion-reperfusion of the left coronary artery, myocardial destruction with focal infiltration of lymphocytes and polymorphonuclear leukocytes was evident in the left ventricular free wall (LVFW) (Figure 1, a and b). Infiltration of inflammatory cells was more diffuse 12 and 24 hours after ischemia/reperfusion (Figure 1c). The infarct lesion in the area was wide 48 hours after reperfusion (Figure 1d). Serum CPK activity was 11-fold higher in infarcted rats than the normal level 3 hours after reperfusion, and it decreased to a threefold higher level at 12 hours (Figure 1e). Therefore, myocardial destruction rapidly progressed after ischemia/reperfusion, and pathological features of the infarct area became apparent by 48 hours after reperfusion.

Plasma HGF levels increased and reached the first peak 3 hours after reperfusion, the level being 6.2-fold higher than that of normal rat plasma (Figure 1f). The level thereafter decreased and reached the second peak at 24 hours, the level being 12.4-fold higher than that of normal (Figure 1f). HGF concentrations in ischemic myocardial tissue 24 hours after reperfusion were also 5.5-fold higher than those of normal rats $-72.2 \pm 10.8$ $\mathrm{ng} / \mathrm{g}$ tissue in ischemic myocardium and $13.6 \pm 2.1$ $\mathrm{ng} / \mathrm{g}$ tissue in normal myocardium, respectively. The cMet/HGF receptor mRNA level in the myocardium was low immediately after ischemia/reperfusion and in sham-operated rats, whereas c-Met mRNA levels markedly increased in the ischemic myocardium of the LVFW, a region affected by ischemic injury, and reached a peak 12 hours after ischemia/reperfusion (Figure 1g). In contrast, upregulation of c-Met mRNA was weak in the myocardium of the interventricular septum (IVS), a nonischemic region. Double immunostaining with c-Met and $\alpha$-sarcomeric actin, a cardiomyocyte-related protein, in infarcted heart tissues also indicated that cardiomyocytes were c-Met-positive in the LVFW adjacent to the infarcted area (Figure $1, \mathrm{~h}$ and i), whereas it was relatively weak in the IVS (Figure $1, \mathrm{j}$ and $\mathrm{k}$ ) and sham-operated heart (Figure 1, part l). Green signals in cardiomyocytes in the border region (Figure $1 \mathrm{~m}$ ) were mostly diminished in control staining with preabsorbed antibody by antigenic peptide (compare Figure $1 \mathrm{~m}$ and inset).

Cardioprotection by HGF in vitro. To determine whether HGF directly exhibits protective actions on cardiomyocytes, we used neonatal rat cardiomyocytes in primary culture. c-Met protein was detectable in cardiomyocytes under normal culture condition, and the c-Met level slightly increased under serum-starved con-

\section{Figure 2}

c-Met expression and cardioprotection by HGF in vitro. (a) c-Met protein expression determined by Western blot. Cardiomyocytes from neonatal rats were cultured for 3 days (lane 1), and further cultured in serum-free condition for 24 hours without (lane 2) or with (lane 3) hydrogen peroxide $\left(\mathrm{H}_{2} \mathrm{O}_{2}\right)$. (b) Survival of cardiomyocytes in culture. The cells were pretreated with HGF for 1 hour, then treated with $50 \mu \mathrm{M} \mathrm{H}_{2} \mathrm{O}_{2}$ for 1 hour and further cultured for 6 hours. ${ }^{A} P<0.05$ vs. $0 \mathrm{ng} / \mathrm{ml}$ of HGF. (c) Activation of ERK-1/2 (p44/p42 mitogen-activated protein kinases) in cultured cardiomyocytes by HGF, as determined by immunoblotting with anti-phospho-ERK-1/2 antibody (top). Immunoblotting with anti-ERK-1 antibody indicates that the total amount of ERK-1/2 protein is similar in two lanes (bottom). (d) Quantification of ERK-1/2 activity. Intensity of the bands was analyzed by densitometry. Each value represents the mean \pm SEM of quadruplicate experiments. a



C

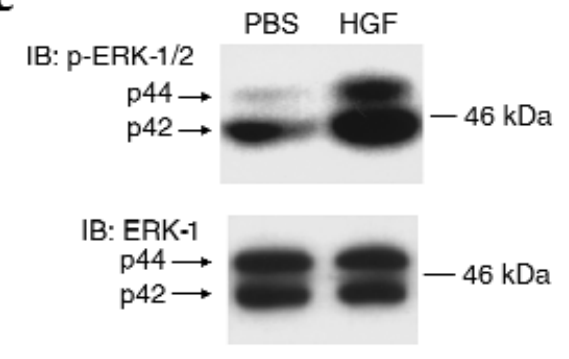

b



d






\section{Figure 3}

Adverse effects of neutralization of endogenous HGF on the ischemia/reperfusion injury model. (a) Specificity of the neutralizing antibody to HGF. Plasma from a rat with ischemia/reperfusion injury was immunoprecipitated with normal IgG (lane 1) or anti-rat HGF IgG (lane 2), and immunoreactive proteins were detected by Western blot, using biotinylated anti-rat HGF IgG. (b) Immunohistochemical staining of infarcted hearts with $\alpha$-sarcomeric actin to depict the infarct area and its quantification. Anti-rat HGF $\operatorname{lgG}(n=10)$ or normal IgG $(n=10)$ was injected 20 minutes before coronary occlusion, and every 12 hours after reperfusion. Forty-eight hours after operation, rats were killed and histological and biochemical analyses were made. Arrowheads indicate the $\alpha$-sarcomeric actin-negative infarct area (original magnification, $\times 40$ ). ${ }^{A} P<0.05$. (c) Change in TUNEL-positive cardiomyocytes by neutralization of endogenous HGF 48 hours after reperfusion. Bars: $100 \mu \mathrm{m}$. ${ }^{\mathrm{B}} P<0.01$. (d) Survival of rats injected with anti-HGF IgG $(n=$ $10)$ or normal $\operatorname{lgG}(n=10)$ after reperfusion. There was a significant difference in survival between the two groups $(P<0.01)$.



c

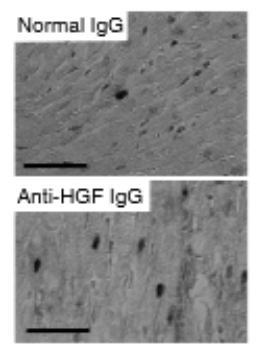

b
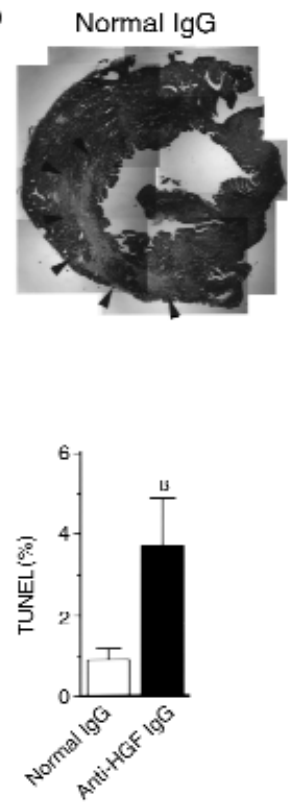


d

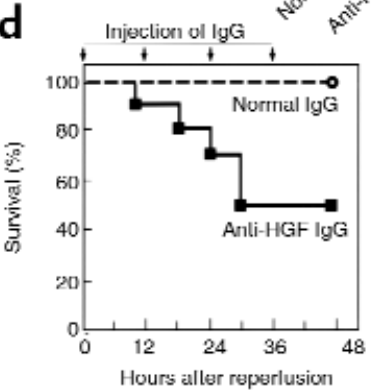

ditions (Figure 2a). We then asked whether HGF would promote the survival of cultured cardiomyocytes. Cardiomyocytes were exposed to $\mathrm{H}_{2} \mathrm{O}_{2}$ to induce oxidative stress in the presence or absence of HGF. Six hours after this treatment, the number of viable cells decreased to $67.9 \%$ of the control. However, HGF dose-dependently increased the number of viable cells, the maximal effect being seen with 30 $\mathrm{ng} / \mathrm{ml} \mathrm{HGF}$ : $95.2 \%$ of cells survived in the presence of $30 \mathrm{ng} / \mathrm{ml}$ HGF (Figure 2b).

Since activation of ERK is closely associated with cytoprotection in cardiomyocytes $(34,35)$, we analyzed ERK-1/2 activation in cultured cardiomyocytes. Under serum-starved conditions, ERK-1/2 was weakly phosphorylated, whereas addition of HGF significantly increased phosphorylated ERK-1/2 (Figure 2, $\mathrm{c}$ and d). HGF dose-dependently activated ERK-1/2, and the maximal effect was seen with 10-30 $\mathrm{ng} / \mathrm{ml}$ HGF (data not shown), similar to the effect on cell survival.

Neutralization of endogenous HGF in ischemia/reperfusion injury. To determine the significance of enhanced expression of HGF and c-Met, we used neutralizing anti-rat HGF IgG $(30,31)$. When plasma from a rat with ischemia/reperfusion injury was immunoprecipitated and subjected to Western blots using the anti-rat HGF IgG, a single band corresponding to HGF was specifically detected (Figure 3a), indicating that this antibody is highly specific to HGF. Five hundred micrograms of the $\operatorname{IgG}$ was given 20 minutes prior to coronary occlusion and every 12 hours after reperfusion. More than $80 \%$ of plasma HGF was complexed with this antibody under this experimental condition (data not shown). The infarct area was immunohistochemically determined to be $\alpha$-sarcomeric actin-negative (Figure $3 b$ ). Infarct area in the group treated with the antibody was significantly larger than that of the control group given normal IgG (Figure 3b). Consistently, HGF neutralization also significantly increased CPK activity in serum from 1410.6 \pm 265.5 to $2023.1 \pm 104.1 \mathrm{IU} / 148$ hours after operation $(P=0.0453)$. TUNEL assay revealed that apoptosis of cardiomyocytes in the LVFW was much higher in the group treated with anti-HGF antibody than in the control group (Figure 3c). Importantly, 50\% of the rats 
injected with anti-HGF IgG died of heart failure within 48 hours after reperfusion, whereas all animals in the control group survived during the period (Figure $3 \mathrm{~d})$. At autopsy, we observed two hearts of animals that died at 12 hours and 18 hours after ischemia/reperfusion; both showed massive myocardial apoptosis, whereas infarction was not obvious at that time (data not shown). Thus apoptosis progresses more rapidly than does necrosis, and this may relate to cardiac dysfunction and death.

We next determined whether plasma from an infarcted rat would promote survival of cardiomyocytes after $\mathrm{H}_{2} \mathrm{O}_{2}$ treatment in vitro. When myocytes from neonatal rats were cultured in the presence of plasma obtained from a normal rat, cell survival decreased to $46.1 \%$ after $\mathrm{H}_{2} \mathrm{O}_{2}$ treatment. On the other hand, the presence of plasma from an infarcted rat promoted sur-

a.
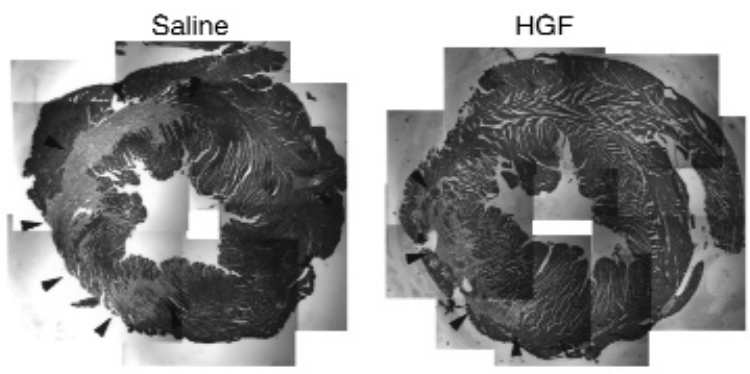

b
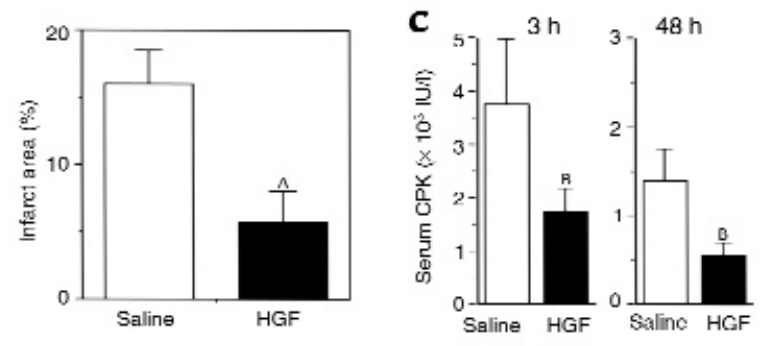

d
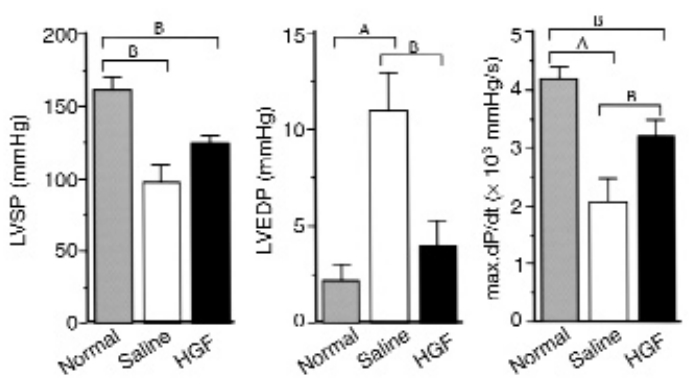

Figure 5

Amelioration of ischemia/reperfusion injury by HGF. Recombinant human $\operatorname{HGF}(n=8)$ or saline $(n=8)$ was injected immediately after and every 12 hours after reperfusion. After 48 hours, rats were killed. (a) $\alpha$-Sarcomeric actin staining done to depict the infarct area (original magnification, $\times 40)$. Arrowheads indicate the $\alpha$-actin-negative infarct area. (b and $\mathbf{c}$ ) Changes in infarct area (b) and serum CPK activity (c). ${ }^{A} P<0.01,{ }^{B} P<0.05$. (d) Change in cardiac functions after ischemia/reperfusion injury. ${ }^{A} P<0.01,{ }^{B} P<0.05$. LVSP, left ventricular systolic pressure; LVEDP, left ventricular end-diastolic pressure; $\max \mathrm{dP} / \mathrm{dt}$, maximal rate of left ventricular pressure rise. vival of the cells to a level similar to that in the control; hence, plasma from an infarcted rat contained endogenous cardioprotective factors. Anti-HGF antibody inhibited the cardioprotective activity in the plasma from an infarcted rat, and $50 \%$ of the activity in the plasma that promoted cell survival was abolished by the anti-HGF antibody. This antibody did not affect survival of the cells exposed to normal plasma (Figure 4). It would thus appear that endogenous HGF is significantly responsible for the cardioprotective effect in the plasma from rats with ischemia/reperfusion injury.

Cardioprotection from myocardial infarction by exogenous HGF. We next addressed the therapeutic potential of HGF in ischemia/reperfusion injury. For administration of HGF, $160 \mu \mathrm{g}$ of recombinant human HGF was given intravenously immediately after reperfusion, and at every 12 hours after reperfusion. In the control group, only the vehicle (saline with $0.1 \% \mathrm{BSA}$ ) was given at the indicated time. Forty-eight hours after reperfusion, the infarct area had extended to $16.0 \%$ of the whole LV in rats not given HGF. In contrast, HGF treatment significantly decreased the infarct area to 5.7\% (Figure 5, a and b). Likewise, HGF administration reduced CPK leakage in serum (Figure 5c). Cardiac function, as evaluated by left ventricular end-diastolic pressure LVEDP and maximal rate of left ventricular pressure rise (maximal $\mathrm{dP} / \mathrm{dt}$ ), was improved by HGF administration to a statistically significant level compared with findings in control saline-treated rats. HGF also improved left ventricular systolic pressure, albeit not statistically (Figure 5d).

In TUNEL staining, the number of TUNEL-positive myocytes was significantly decreased by HGF-treatment (Figure 6a). Bcl-xL was specifically detected in myocytes in the ischemic area as early as 3 hours after the treatment (Figure 6b), but not in the nonischemic area (data not shown). Of note, Bcl-xL expression in cardiomyocytes was increased by HGF administration (Figure 6b). Consistently, Western blot analysis of Bcl-xL levels in myocardial tissue extracts indicated that Bcl-xL expression was slightly increased by ischemia/reperfusion injury and that HGF treatment significantly increased it to a much higher level than in control myocardial tissue (Figure 6c). Bcl-2 was undetectable under these experimental conditions (data not shown). Similar to these changes, Bcl-xL expression was undetectable in cardiomyocytes cultured under normal conditions but was detectable 12 hours after serum-starvation. On the other hand, Bcl-xL expression was rapidly induced upon HGFtreatment 1 hour after serum-starvation and further increased to a much higher level than that in control cultures (Figure 6d). Therefore, the cardioprotective action of HGF is due, in part, to a direct antiapoptotic effect on cardiomyocytes.

\section{Discussion}

Our data support the notion that HGF has a definite role in cardioprotection from ischemia/reperfusion injury as an endogenous cardiotrophic factor, and that this cardioprotection is exerted through the direct action of 
a

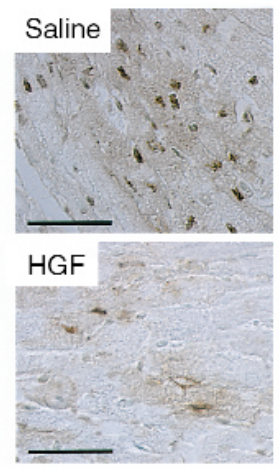

C

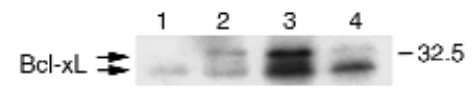

b
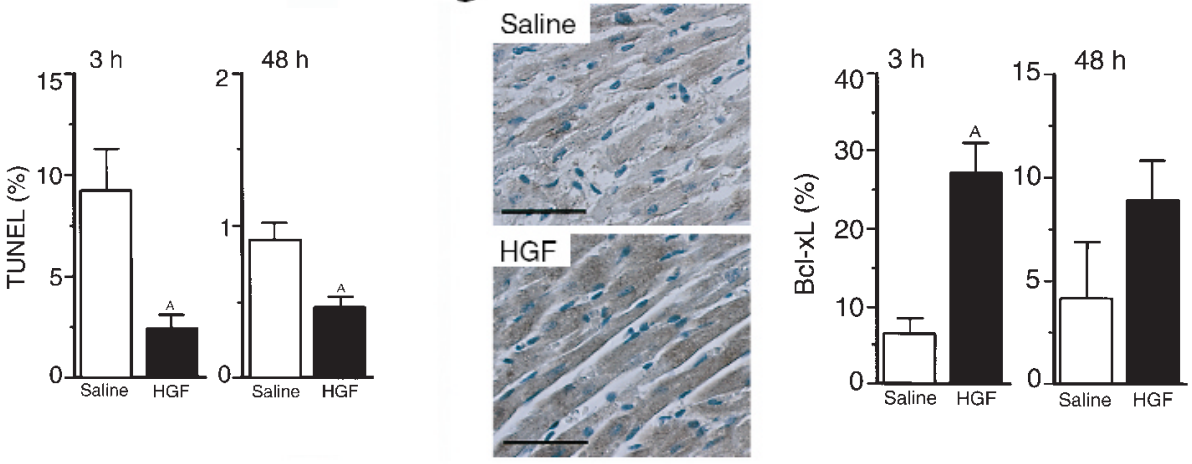

d

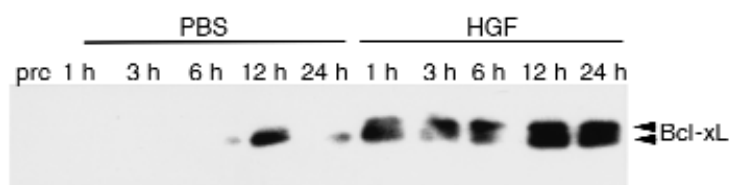

\section{Figure 6}

Antiapoptotic effect of HGF on the ischemia/reperfusion injury model. Hearts were excised 3 hours and 48 hours after reperfusion. (a) Distribution ofTUNEL-positive cardiomyocytes 3 hours after reperfusion (bars: $100 \mu \mathrm{m}$ ) and changes in TUNEL-positive cells by HGF-treatment. ${ }^{A} P$ $<0.05$. (b) Immunohistochemical staining for $\mathrm{Bcl}-\mathrm{xL}$ in the rat heart and semiquantitative analysis (bars: $100 \mu \mathrm{m}$ ). Bar graphs represent percent of $\mathrm{Bcl}$-xL-positive myocytes observed at least in ten fields per section. ${ }^{A} P<0.05$. (c) Change in $\mathrm{Bcl}$-xL expression in myocardial extracts detected by Western blot. Lane 1, sham-operated LV; lane 2, ischemia/reperfused LV with saline treatment; lane 3, ischemia/reperfused LV with HGF-treatment; lane 4, normal rat spleen for positive control. (d) Bcl-xL expression in cardiomyocytes in culture. Cardiomyocytes were cultured in serum-free medium in the presence or absence of HGF for the indicated period. Expression of Bcl-xL was detected by Western blot.

HGF on cardiomyocytes. Evidence for this proposal includes: (a) the neutralization of HGF in vivo resulted in exacerbated pathology and increased mortality due to cardiac failure; (b) HGF directly exhibited protective effects on cardiomyocytes in vitro, and the cardioprotective activity in the plasma from rats with induced ischemia/reperfusion injury was significantly suppressed by the neutralization of HGF; (c) both c-Met/HGF receptor expression in cardiomyocytes and plasma HGF levels rapidly increased in response to ischemia/reperfusion injury; and (d) administration of HGF to rats with ischemia/reperfusion injury resulted in a much lesser degree of myocardial apoptosis and infarct area, and better cardiac function, compared with control rats.

It is noteworthy that serum HGF levels in patients with myocardial infarction were over 50 times higher than those in healthy humans, but the biological significance of this remains to be addressed (26). Our results suggest that the increased blood HGF may reflect defensive reactions and participate in cardioprotection against myocardial infarction, possibly also in humans. Although the source of plasma HGF that increased following ischemia/reperfusion injury remains to be defined, rapid induction of HGF mRNA expression was seen in distant intact organs such as the liver, kidney, lung, and spleen, as well as in the injured heart after ischemia/reperfusion injury in rats (ref. 36, and our unpublished data), suggesting that endogenous HGF might be derived from these organs. A similar rapid induction of HGF expression in injured and distant intact organs was noted in the case of acute hepatic and renal injuries $(37,38)$, and the presence of a humoral factor that induces HGF expression was noted in plasma of rats with hepatic and renal injuries (39). In addition to an increase in HGF levels in plasma, there was also rapid upregulation of c-Met receptor after ischemia/reperfusion injury. Since biological effects of HGF on cardiomyocytes, as revealed by serum $\mathrm{CPK}, \mathrm{Bcl}-\mathrm{xL}$ expression, and myocardial apoptosis, were seen as early as 3 hours after reperfusion, c-Met receptor expressed in normal cardiomyocytes at a relatively low level might be responsible for early signaling to protect the myocardium. Subsequent increase in c-Met mRNA may possibly relate to the autoinduction of gene expression triggered by $\operatorname{HGF}(38,40)$.

Myocardial apoptosis occurs after ischemia/reperfusion injury (2-4), and prevention of it has gained much attention as a new target for cardioprotection from ischemia/reperfusion injury $(41,42)$. We found that biochemical neutralization of HGF resulted in increased apoptosis, while HGF administration resulted in a much-decreased apoptosis in cardiomyocytes, and HGF was cytoprotective for cultured cardiomyocytes. Therefore, the cardioprotective effects of HGF may be mainly attributable to its antiapoptotic action on cardiomyocytes. With respect to mechanisms that relate to the antiapoptotic effects of HGF, we focused on Bcl-xL expression in cardiomyocytes, since Bcl-xL has a critical role in antiapoptosis, counteracting apoptotic signals in a variety of cells, including car- 
diomyocytes (43). In addition, previous studies showed that prevention of massive hepatocyte apoptosis and fulminant hepatic failure by HGF were associated with a remarkable induction of $\mathrm{Bcl}-\mathrm{xL}(23,24)$. Therefore, the rapid induction of $\mathrm{Bcl}-\mathrm{xL}$ expression in cardiomyocytes may be involved in the antiapoptosis achieved by HGF. On the other hand, although we focused here on the antiapoptotic action of HGF, necrotic cell death after myocardial infarction has to be considered. Recent studies revealed that intracellular signaling pathways leading to antiapoptotic and antinecrotic cell death are at least partially shared (44). Overexpression of Bcl-2 in hepatocytes suppressed necrotic cell death (45). Moreover, activation of ERK-1/2 participates in the reduction of necrotic cell death, as well as apoptotic cell death (46). Therefore, rapid induction of Bcl-xL and activation of ERK by HGF in cardiomyocytes make way for the notion that HGF may give a signal to reduce necrosis in cardiomyocytes following myocardial infarction.

Other researchers reported that protective effects of cardiotrophic factors such as IGF-1 and leukemia inhibitory factor involve ERK activation $(47,48)$. Inhibition of ERK phosphorylation by a specific inhibitor resulted in increased apoptosis in cardiomyocytes (49) and ischemia/reperfusion injury in isolated rat hearts (34). In addition to the ERK-related pathway, stimulation of Akt downstream of phosphatidylinositol (PI) 3kinase is involved in protection of cardiomyocytes by IGF-1 (50). However, HGF did not induce Akt phosphorylation; thus the PI 3-kinase-Akt pathway does not seem to be involved in HGF-dependent antiapoptosis in cardiomyocytes (our unpublished data). Therefore, the intracellular signaling pathways leading to antiapoptosis in cardiomyocytes by HGF and to that by IGF-1 differ, at least in part, although HGF and IGF-1 exhibit similar cardioprotective actions, some of which are mediated by receptor tyrosine kinases. In this context, it is noteworthy that Bag- 1 , a partner of $\mathrm{Bcl}-2 / \mathrm{Bcl}-\mathrm{xL}$, is specifically associated with the c-Met receptor. Association of $\mathrm{Bag}-1$ with $\mathrm{Bcl}-2 / \mathrm{Bcl}-\mathrm{xL}$ enhances antiapoptotic action of $\mathrm{Bcl}-2 / \mathrm{Bcl}-\mathrm{xL}$, and expression of Bag- 1 in cells promotes the cell survival activity of HGF (51).

Although the cardioprotective and antiapoptotic effects of HGF on cardiomyocytes are likely to be largely attributable to direct actions on cardiomyocytes, previous studies revealed a potent angiogenic action of HGF in vivo $(52,53)$. Likewise, transfection of HGF gene into the rat myocardium resulted in a significant increase in the number of blood vessels (54). Together with findings that administration or gene transfection of angiogenic growth factors into laboratory animals promoted coronary angiogenesis and resulted in a diminished pathology due to myocardial infarction $(55,56)$, biological actions of HGF for both myocardium and coronary vessels may further minimize the extensive myocardial injury due to prolonged ischemia.

In summary, endogenous and exogenous HGF protected the rat heart against ischemia/reperfusion injury.
Preclinical and clinical trials of HGF for treatment of patients with myocardial infarction can be considered.

\section{Acknowledgments}

We are grateful to $\mathrm{M}$. Ohara for assistance in revising this manuscript and H. Ueda (Osaka University Graduate School of Medicine) for technical support. This work was supported by a grant from the Ministry of Education, Science, Sports, and Culture of Japan, a research grant from the Japan Heart Foundation, and an IBM Japan Research Grant.

1. Lopez, A.D., and Murray, C.C. 1998. The global burden of disease, 1990-2020. Nat. Med. 4:1241-1243.

2. Gottlieb, R.A., et al. 1994. Reperfusion injury induces apoptosis in rabbit cardiomyocytes. J. Clin. Invest. 94:1621-1628.

3. Fliss, H., and Gattinger, D. 1996. Apoptosis in ischemic and reperfused rat myocardium. Circ. Res. 79:949-956.

4. Bialik, S., et al. 1997. Myocyte apoptosis during acute myocardial infarction in the mouse localizes to hypoxic regions but occurs independently of p53. J. Clin. Invest. 100:1363-1372.

5. Colucci, W.S. 1996. Apoptosis in the heart. N. Engl. J. Med. 335:1224-1226.

6. Nakamura, T., Nawa, K., and Ichihara, A. 1984. Partial purification and characterization of hepatocyte growth factor from serum of hepatectomized rats. Biochem. Biophys. Res. Commun. 122:1450-1459.

7. Nakamura, T., et al. 1989. Molecular cloning and expression of human hepatocyte growth factor. Nature. 342:440-443.

8. Zarnegar, R., and Michalopoulos, G.K. 1995. The many faces of hepatocyte growth factor: from hepatopoiesis to hematopoiesis. J. Cell Biol. 129:1177-1180.

9. Matsumoto, K., and Nakamura, T. 1996. Emerging multipotent aspects of hepatocyte growth factor. J. Biochem. 119:591-600.

10. Kopp, J.B. 1998. Hepatocyte growth factor: mesenchymal signal for epithelial homeostasis. Kidney Int. 54:1392-1393.

11. Balkovetz, D.F., and Lipschutz, J.H. 1999. Hepatocyte growth factor and the kidney: it is not just for the liver. Int. Rev. Cytol. 186:225-260.

12. Garcia-Ocana, A., et al. 2000. Hepatocyte growth factor overexpression in the islet of transgenic mice increases beta cell proliferation, enhances islet mass, and induces mild hypoglycemia. J. Biol. Chem. 275:1226-1232.

13. Bottaro, D.P., et al. 1991. Identification of the hepatocyte growth factor receptor as the c-met proto-oncogene product. Science. 251:802-804

14. Naldini, L., et al. 1991. Hepatocyte growth factor (HGF) stimulates the tyrosine kinase activity of the receptor encoded by the proto-oncogene c-MET. Oncogene. 6:501-504.

15. Ishiki, Y., Ohnishi, H., Muto, Y., Matsumoto, K., and Nakamura, T. 1992. Direct evidence that hepatocyte growth factor is a hepatotrophic factor for liver regeneration and has a potent antihepatitis effect in vivo. Hepatology. 16:1227-1235.

16. Michalopoulos, G.K., and DeFrances, M.C. 1997. Liver regeneration. Science. 276:60-66.

17. Kawaida, K., Matsumoto, K., Shimazu, H., and Nakamura, T. 1994. Hepatocyte growth factor prevents acute renal failure and accelerates renal regeneration in mice. Proc. Natl. Acad. Sci. USA. 91:4357-4361.

18. Mizuno, S., et al. 1998. Hepatocyte growth factor prevents renal fibrosis and dysfunction in a mouse model of chronic renal disease. J. Clin. Invest. 101:1827-1834.

19. Ohmichi, H., Matsumoto, K., and Nakamura, T. 1996. In vivo mitogenic action of HGF on lung epithelial cells: pulmotrophic role in lung regeneration. Am. J. Physiol. 270:L1031-L1039.

20. Panos, R.J., Patel, R., and Bak, P.M. 1996. Intratracheal administration of hepatocyte growth factor/scatter factor stimulates rat alveolar type II cell proliferation in vivo. Am. J. Respir. Cell. Mol. Biol. 15:574-581.

21. Liu, Y., Sun, A.M., and Dworkin, L.D. 1998. Hepatocyte growth factor protects renal epithelial cells from apoptotic cell death. Biochem. Biophys. Res. Commun. 246:821-826.

22. Maina, F., and Klein, R. 1999. Hepatocyte growth factor, a versatile signal for developing neurons. Nat. Neurosci. 2:213-217.

23. Kosai, K., Matsumoto, K., Nagata, S., Tsujimoto, Y., and Nakamura, T. 1998. Abrogation of Fas-induced fulminant hepatic failure in mice by hepatocyte growth factor. Biochem. Biophys. Res. Commun. 244:683-690

24. Kosai, K., Matsumoto, K., Funakoshi, H., and Nakamura, T. 1999. Hepatocyte growth factor prevents endotoxin-induced lethal hepatic failure in mice. Hepatology. 30:151-159. 
25. Rappolee, D.A., Iyer, A., and Patel, Y. 1996. Hepatocyte growth factor and its receptor are expressed in cardiac myocytes during early cardiogenesis. Circ. Res. 78:1028-1036.

26. Matsumori, A., et al. 1996. Increased circulating hepatocyte growth factor in the early stage of acute myocardial infarction. Biochem. Biophys. Res. Commun. 221:391-395.

27. Ueda, H., et al. 1999. Gene transfection of hepatocyte growth factor attenuates reperfusion injury in the heart. Ann. Thorac. Surg. 67:1726-1731.

28. Buerke, M., et al. 1995. Cardioprotective effect of insulin-like growth factor I in myocardial ischemia followed by reperfusion. Proc. Natl. Acad. Sci. USA. 92:8031-8035.

29. Depre, C., et al. 1998. Unloaded heart in vivo replicates fetal gene expression of cardiac hypertrophy. Nat. Med. 4:1269-1275.

30. Santos, O.F., et al. 1994. Involvement of hepatocyte growth factor in kidney development. Dev. Biol. 163:525-529.

31. Ohmichi, H., Koshimizu, U., Matsumoto, K., and Nakamura, T. 1998. Hepatocyte growth factor (HGF) acts as a mesenchyme-derived morphogenic factor during fetal lung development. Development. 125: $1315-1324$.

32. Li, Q., et al. 1997. Overexpression of insulin-like growth factor-1 in mice protects from myocyte death after infarction, attenuating ventricular dilation, wall stress, and cardiac hypertrophy. J. Clin. Invest. 100:1991-1999.

33. Simpson, P., McGrath, A., and Savion, S. 1982. Myocyte hypertrophy in neonatal rat heart cultures and its regulation by serum and by catecholamines. Circ. Res. 51:787-801.

34. Yue, T.L., et al. 2000. Inhibition of extracellular signal-regulated kinase enhances ischemia/reoxygenation-induced apoptosis in cultured cardiac myocytes and exaggerates reperfusion injury in isolated perfused heart. Circ. Res. 86:692-699.

35. Adderley, S.R., and Fitzgerald, D.J. 1999. Oxidative damage of cardiomyocytes is limited by extracellular regulated kinases $1 / 2$-mediated induction of cyclooxygenase-2. J. Biol. Chem. 274:5038-5046.

36. Ono, K., Matsumori, A., Shioi, T., Furukawa, Y., and Sasayama, S. 1997. Enhanced expression of hepatocyte growth factor/c-Met by myocardial ischemia and reperfusion in a rat model. Circulation. 95:2552-2558.

37. Kono, S., Nagaike, M., Matsumoto, K., and Nakamura, T. 1992. Marked induction of hepatocyte growth factor mRNA in intact kidney and spleen in response to injury of distant organs. Biochem. Biophys. Res. Commun. 186:991-998.

38. Liu, Y., et al. 1999. Up-regulation of hepatocyte growth factor receptor: an amplification and targeting mechanism for hepatocyte growth factor action in acute renal failure. Kidney Int. 55:442-453.

39. Matsumoto, K., et al. 1992. Identification and characterization of "injurin," an inducer of expression of the gene for hepatocyte growth factor. Proc. Natl. Acad. Sci. USA. 89:3800-3804.

40. Boccaccio, C., Gaudino, G., Gambarotta, G., Galimi, F., and Comoglio, P.M. 1994. Hepatocyte growth factor (HGF) receptor expression is inducible and is part of the delayed-early response to HGF. J. Biol.
Chem. 269:12846-12851.

41. Yaoita, H., Ogawa, K., Maehara, K., and Maruyama, Y. 1998. Attenuation of ischemia/reperfusion injury in rats by a caspase inhibitor. Circulation. 97:276-281.

42. Vakeva, A.P., et al. 1998. Myocardial infarction and apoptosis after myocardial ischemia and reperfusion: role of the terminal complement components and inhibition by anti-C5 therapy. Circulation. 97:2259-2267.

43. Olivetti, G., et al. 1997. Apoptosis in the failing human heart. N. Engl. J. Med. 336:1131-1141.

44. Tsujimoto, Y., Shimizu, S., Eguchi, Y., Kamiike, W., and Matsuda, H. 1997. Bcl-2 and Bcl-xL block apoptosis as well as necrosis: possible involvement of common mediators in apoptotic and necrotic signal transduction pathways. Lenkemia. 11(Suppl. 3):380-382.

45. Yamabe, K., et al. 1998. Prevention of hypoxic liver cell necrosis by in vivo human bcl-2 gene transfection. Biochem. Biophys. Res. Commun. 243:217-223.

46. di Mari, J.F., Davis, R., and Safirstein, R.L. 1999. MAPK activation determines renal epithelial cell survival during oxidative injury. Am. J. Physiol. 277:F195-F203.

47. Foncea, R., et al. 2000. Extracellular regulated kinase, but not protein kinase $\mathrm{C}$, is an antiapoptotic signal of insulin-like growth factor- 1 on cultured cardiac myocytes. Biochem. Biophys. Res. Commun. 273:736-744.

48. Kunisada, K., et al. 1996. Activation of JAK-STAT and MAP kinases by leukemia inhibitory factor through gp130 in cardiac myocytes. Circulation. 94:2626-2632.

49. Zhu, W., et al. 1999. MAPK superfamily plays an important role in daunomycin-induced apoptosis of cardiac myocytes. Circulation. 100:2100-2107.

50. Fujio, Y., et al. 2000. Akt promotes survival of cardiomyocytes in vitro and protects against ischemia/reperfusion injury in mouse heart. Circulation. 101:660-667.

51. Bardelli, A., et al. 1996. HGF receptor associates with the anti-apoptotic protein BAG-1 and prevents cell death. EMBO J. 15:6205-6212.

52. Grant, D.S., et al. 1993. Scatter factor induces blood vessel formation in vivo. Proc. Natl. Acad. Sci. USA. 90:1937-1941.

53. Van Belle, E., et al. 1998. Potentiated angiogenic effect of scatter factor/hepatocyte growth factor via induction of vascular endothelial growth factor: the case for paracrine amplification of angiogenesis. Circulation. 97:381-390

54. Aoki, M., et al. 2000. Angiogenesis induced by hepatocyte growth factor in non-infarcted myocardium and infarcted myocardium: up-regulation of essential transcription factor for angiogenesis, ets. Gene Ther. 7:417-727.

55. Yanagisawa-Miwa, A., et al. 1992. Salvage of infarcted myocardium by angiogenic action of basic fibroblast growth factor. Science. 257:1401-1403.

56. Giordano, F.J., et al. 1996. Intracoronary gene transfer of fibroblast growth factor-5 increases blood flow and contractile function in an ischemic region of the heart. Nat. Med. 2:534-539. 